[0212-7199 (2005) 22: 7; pp 323-325] ANALES DE MEDICINA INTERNA Copyright $@ 2005$ ARAN EDICIONES, S.L.

AN. MED. INTERnA (Madrid) Vol. 22, N. ${ }^{\circ}$, pp. 323-325, 2005

\section{Relación entre la ingesta de ácidos grasos omega 3 y los valores de CD4 en pacientes con infección por VIH: un estudio transversal}

\author{
D. A. DE LUIS, P. BACHILLER ${ }^{1}$, O. IZAOLA, J. M. EIROS BOUZA
}

Sección de Endocrinología y Nutrición Clínica. Hospital del Río Hortega. ${ }^{1}$ Instituto de Endocrinología y Nutrición Clínica. Facultad de Medicina. Hospital Clínico Universitario. Valladolid

\title{
RESUMEN
}

Objetivos: Los ácidos grasos poliinsaturados, son agentes inmunomoduladores. Estos nutrientes podrían tener un papel relevante en los pacientes con infección por VIH. El objetivo de nuestro trabajo fue analizar la relación entre la ingesta de ácidos grasos poliinsaturados y los niveles de linfocitos CD4 en estos pacientes.

Pacientes y métodos: Un total de 40 pacientes con infección por VIH fueron estudiados. En todos los pacientes se registraron las siguientes variables; edad, sexo, tratamiento antiretroviral y se realizó una evaluación antropométrica (peso, pliegue tricipital, circunferencia muscular, índice de masa corporal (IMC)), así como una valoración bioquímica (albúmina, prealbúmina, transferrina, proteínas totales, linfocitos totales y recuento de CD4). A todos los pacientes se les realizó una encuesta nutricional de 3 días.

Resultados: La edad media de los 40 pacientes (24 varones y 16 mujeres) fue de $37,6 \pm 9,4$ años, peso $65,6 \pm 12,2 \mathrm{~kg}$ e índice de masa corporal $23,2 \pm 3,25$. La evaluación antropométrica mostró un valor medio del pliegue tricipital de $15,1 \pm 5,3 \mathrm{~mm}$, circunferencia muscular del brazo $27,5 \pm 3,1 \mathrm{~cm}$. La ingesta calórica y la distribución de macronutrients fue normal (carbohidratos $48 \%$, proteínas 18\% y lípidos $34 \%$ ). La ingesta de ácidos grasos poliinsaturados fue la siguiente; ácidos omega 3 (1,22 $\pm 3,1 \mathrm{~g} /$ día), ácidos omega $6(5,76 \pm 4,6 \mathrm{~g} /$ día $)$ y ácidos omega 9 (31,5 $\pm 11 \mathrm{~g} /$ día). En el análisis multivariante, con los niveles de CD4 como variable dependiente, mostró como variable independiente los niveles de omega $3(\mathrm{~F}=6,2 ; \mathrm{p}<0,05)$, con un incremento de $32 \mathrm{CD} 4$ (IC95\%: 9,86-52,14) CD4+ (recuento/uL) por cada $1 \mathrm{~g}$ de ácidos omega 3 ajustado por edad, sexo y fármacos antiretrovirales.

Conclusión: En pacientes con infección por VIH, la ingesta de ácidos grasos omega 3 se correlaciona con un incremento de los valores de CD4.

PALABRAS CLAVE: Ácidos grasos poliinsaturados. Infección por VIH.

De Luis DA, Bachiller P, Izaola O, Eiros Bouza JM. Relación entre la ingesta de ácidos grasos omega 3 y los valores de CD4 en pacientes con infección por VIH: un estudio transversal. An Med Interna (Madrid) 2005; 22: 323-325.

\section{INTRODUCCIÓN}

La relación entre la infección por el virus de la inmunodeficiencia humana (VIH), la nutrición y la función inmune es
RELATION BETWEEN INTAKE OF OMEGA 3 FATTY ACIDS AND CD4 COUNT IN PATIENTS WITH HIV INFECTION: A TRANSVERSAL STUDY

\section{ABSTRACT}

Objectives: Polyunsaturated fatty acids are immunomodulatory agents. These agents could develop a main role in patients with HIV infection. The aim of our study was to analyse the relation between polyunsaturated fatty acid intake and CD4 count in HIV infected patients.

Patients and methods: Forty patients with HIV infections were enrolled. In all patients, the next variables were recorded; age, sex, drugs, anthropometric evaluation (weight, height, tricipital skinfold, midarm circumference, body mass index) and a biochemical evaluation (albumin, prealbumin, trasferrin, total proteins, total lymphocytes and CD4 count). A 3 day dietary intake questionnaire was performed, too.

Results: The mean age was (24 males and 16 females) $37.6 \pm 9.4$ years, mean weight $65.6 \pm 12.2 \mathrm{~kg}$ and body mass index $23.2 \pm 3.25$. Anthropometric evaluation showed a mean tricipital skinfold of $15.1 \pm$ $5.3 \mathrm{~mm}$, midarm circumference $27.5 \pm 3.1 \mathrm{~cm}$. Caloric intake and macronutrient distribution were normal (carbohydrates $48 \%$, proteins $18 \%$ and lipids $34 \%$ ). Dietary intake of polyunsaturated fatty acids was omega $3(1.22 \pm 3.1 \mathrm{~g} /$ day $)$ and omega $9(31.5 \pm 11 \mathrm{~g} /$ day $)$. Multivariante analysis, with CD4 count as a dependent variable, showed omega 3 intake as a independent variable $(F=6.2 ; p<0.05)$, with an increase of 32 (IC95\%: 9.86-52.14) CD4+ (count/uL) with each $1 \mathrm{~g}$ of omega 3 dietary intake adjusted by age, sex, and drugs.

Conclusion: In patients with HIV infection, dietary intake of omega 3 fatty acids increase CD4 count.

KEY WORDS: Polyunsaturated fatty acids. HIV infection.

\section{Trabajo aceptado: 5 de abril de 2005}

Correspondencia: Daniel de Luis Román. Profesor Asociado de Endocrinología y Nutrición. Director Ejecutivo IEN. Facultad de Medicina. Hospital Universitario Río Hortega. C/ Perales, 16. 47103 Valladolid. e-mail: dadluis@yahoo.es 
Los ácidos grasos omega 3 han mostrado en diferentes patologías un efecto inmunomodulador, modulando la respuesta inflamatoria. No obstante los trabajos que evalúan estos efectos son escasos (3).

El objetivo del estudio fue evaluar la relación de la ingesta de ácidos grasos poliinsaturados y el recuento de CD4.

\section{PACIENTES Y MÉTODOS}

Un total de 40 pacientes con VIH fueron evaluados. Los criterios de inclusión fueron; infección por VIH confirmada, ausencia de proceso febril crónico, ausencia de síntomas gastrointestinales (diarrea más de 30 día o más de 3 veces al día), función hepática y renal adecuada. Se excluyeron los pacientes que tomaban suplementos nutricionales. En todos los pacientes se registraron las siguientes variables; edad, sexo, tratamiento antiretroviral y se realizó una evaluación antropométrica (peso, pliegue tricipital, circunferencia muscular, índice de masa corporal (IMC)). Todos los pacientes dieron un consentimiento informado y permanecieron con su terapia antiretroviral habitual.

Evaluación nutricional. Se determinó el índice de masa corporal $(\mathrm{IMC}=$ peso/talla 2$)$. El peso fue determinado en una báscula calibrada en $100 \mathrm{~g}$ y la talla en un tallímetro en bipedestación calibrado en mm. El pliegue tricipital y la cicunferencia muscular del brazo fue determinado de una manera estándar $(4,5)$.

Evaluación bioquímica. Se realizó una extracción basal de sangre para determinar el recuento de CD4 (LabCorp, Unionandlae, NY), así como los niveles de albúmina (3,5-4,5 g/dl), prealbúmina $(18-28 \mathrm{mg} / \mathrm{dl})$, transferían $(250-350 \mathrm{mg} / \mathrm{dl})$, y linfocitos $(1.200-3.500 .103 / \mathrm{mL}$ ) con el autoanalizador (Hitachi, ATM, Manheim, Ger).

Ingesta dietética. Todos los pacientes recibieron instrucciones para cumplimentar una encuesta nutricional de 3 días, incluyendo un día de fin de semana. Las encuestas fueron calibradas por una dietista, utilizando un software propio de la Unidad, con las tablas nacionales de composición de alimentos como referencia (6).

Análisis estadístico. Los resultados se expresaron como media \pm desviación estandar. La distribución de las variables fue analizada con el test de Kolmogorov-Smirnov. Las pruebas de Pearson y Spearman fueron utilizadas en el análisis univariante de correlación. Se realizó un análisis multivariante, utilizando como variable dependiente (CD4), e introduciendo en el modelo las variables estadisticamente significativas del análisis univariante. Un valor de $\mathrm{p}<0,05$ fue considerado estadísticamente significativo.

\section{RESULTADOS}

Un total de 40 pacientes infectados por VIH (24 varones y 16 mujeres) fueron estudiados, con una edad media de 37,6 \pm 9,4 años, peso $65,6 \pm 12,2 \mathrm{Kg}$ e IMC 23,2 $\pm 3,25$. Todos los pacientes estaban recibiendo terapia antiretroviral, un $5 \%$ de los pacientes con 2 fármacos, un $75 \%$ con 3 fármacos y un $20 \%$ con 4 fármacos. La distribución de los pacientes en los diferentes estadios de la infección fue $25 \%$ estadio A, $37,5 \%$ estadio B y $37,5 \%$ estadio C (7).
La tabla I muestra los parámetros bioquímicos y antropométricos de los pacientes. La evaluación antropométrica mostró unos valores medios del pliegue tricipital $15,1 \pm 5,3 \mathrm{~mm}$ y la circunferencia muscular del brazo $27,5 \pm 3,1 \mathrm{~cm}$.

\section{TABLA I}

\begin{tabular}{lcc}
\multicolumn{3}{c}{ TABLA I } \\
\hline PARÁMETROS BIOQUÍMICOS \\
\hline Parámetros & Medias (SD) & Valores normales \\
\hline Proteínas totales $(\mathrm{g} / \mathrm{dl})$ & $7,8 \pm 0,6$ & $(6-8)$ \\
Albúmina $(\mathrm{g} / \mathrm{dl})$ & $4,6 \pm 2,9$ & $(3-5)$ \\
Prealbúmina $(\mathrm{mg} / \mathrm{dl})$ & $27,1 \pm 20$ & $(20-30)$ \\
Transferrina $(\mathrm{mg} / \mathrm{dl})$ & $245 \pm 62$ & $(200-350)$ \\
Linfocitos $\left(10^{3} / \mathrm{uL}\right)$ & $2.420 \pm 720$ & $(2.000-3.500)$ \\
CD4 (recuento $/ \mathrm{uL})$ & $552,8 \pm 273$ & $(>400)$ \\
\hline
\end{tabular}

La tabla II muestra la ingesta de macronutrientes comparado con las recomendaciones internacionales (8). La ingesta calórica total y la distribución de macronutrients fue normal (carbohidratos 48\%, proteínas 18\% y lípidos 34\%). La ingesta media de ácidos grasos poliinsaturados fue la siguiente; $1,22 \pm 3,1 \mathrm{~g}$ /día de ácidos omega 3, 5,76 \pm 4,6 g/día de ácidos omega 6 y 31,5 $\pm 11 \mathrm{~g} /$ día de ácidos omega 9 . La relación omega 6/omega3 fue de $4,7 \pm 2,8$. No existieron diferencias estadisticamente significativas al analizar la ingesta de macronutrientes y ácidos grasos en función del estadio de la enfermedad o del número de fármacos antiretrovirales que tomaban los pacientes.

\section{TABLA \|}

PORCENTAJE DE LAS RDA EN LOS PACIENTES CON INFECCIÓN POR VIH

\begin{tabular}{lcc}
\hline & Media (SD) & Porcentaje de RDA \\
\hline Macronutrientes: & & \\
$\quad$ Carbohidratos (g/día) & $216,8 \pm 96,3$ & - \\
Lipidos (g/día) & $75,3 \pm 27,1$ & - \\
Proteínas (g/día) & $81,6 \pm 24,9$ & - \\
Calorías (cal/día) & $1786 \pm 475$ & - \\
C de proteínas/kg & $1,27 \pm 0,4$ & $170 \%$ \\
Calorías/kg & $28,1 \pm 8,1$ & - \\
\hline
\end{tabular}

El análisis de correlación entre la ingesta de nutrientes y lípidos y los niveles de CD4 mostró una correlación positiva la ingesta de ácidos poliinsaturados omega 3 y las calorías con el recuento de CD4, $(r=0,45 ; p<0,05)$ y $(r=-0,25 ; p$ $<0,05)$, respectivamente. En el análisis multivariante, con la variable dependiente (recuento de CD4), solo la ingesta de ácidos grasos omega 3 permaneció en el modelo $(\mathrm{F}=6,2 ; \mathrm{p}$ $<0,05$ ), con un incremento de 32 (IC95\%: 9,86-52,14) CD4+ (recuento/uL) por cada incremento de la ingesta de 1 $\mathrm{g}$ de ácidos omega 3, ajustado por edad, sexo y numero de drogas. 


\section{DISCUSIÓN}

Una gran cantidad de estudios han mostrado la asociación entre la progresión de la infección por VIH y la ingesta dietética. En nuestra población de pacientes con infección por VIH la distribución de macronutrientes de la dieta que seguían fue normal. Este dato puede explicar el peso e índice de masa corporal que presentaban los pacientes. No obstante, los requerimientos proteicos de estos pacientes pueden estar modificadas debido a un aumento de la tasa de infecciones oportunistas de esta población, con el consiguiente incremento del turnover proteico (10-13).

Nuestro grupo mostró en un trabajo previo (3), un incremento en el recuento de CD4 y del peso en una población de pacientes con infección VIH al suplementar su dieta con una fórmula enriquecida de ácidos grasos omega 3. Unos resultados similares fueron encontrados en otro trabajo (14), no obstante este efecto no se sabe si es secundario al incremento de la ingesta de estos ácidos o al incremento en la ingesta proteica o energética. Sin embargo, se ha demostrado que los ácidos omega 3 son incorporados en las membranas celulares, remplazando el ácido araquidónico, el cual deriva de los ácidos omega 6. Esta sustitución en membranas es menos inflamato- ria, generando una menor producción de citokinas (14). Además, la presencia de ácidos omega 3 en la membrana reduce la producción de eicosanoides inmunosupresores (e.g., prostaglandinas de la serie 2, leukotrienos de la serie 4 y tromboxanos de la serie 2).

Como podemos observar la relación de la ingesta de ácidos omega 3 con beneficios inmunomoduladores, parece más o menos clara, no obstante los trabajos comentados hacen referencia a la utilización de suplementos enriquecidos en ácidos omega 3. Nuestro trabajo muestra como la alimentación oral sin suplementar puede modular la situación inmunológica de esta población. No obstante la utilización de suplementos nutricionales en pacientes con infección VIH, ha mostrado su superioridad que la simple educación nutricional (15), por tanto son necesarios más estudios descriptivos que muestre la situación nutricional y de ingesta de estos pacientes (16-18) y futuros estudios de intervención que ayuden a aclarar los efectos beneficiosos de los diferentes suplementos existentes.

En conclusión, la ingesta diaria de ácidos grasos poliinsaturados omega 3 se correlaciona de manera positiva con los niveles de CD4 de una muestra de pacientes con infección por VIH.

\section{Bibliografía}

1. Guenter P, Muurahein N, Simons G, Kosos KA, Cohan Gr, Rudenstein $\mathrm{R}$, Turner JL. Relationship between nutritional status, disease progression, and survival in HIV infection. J Acquir Immune Defic Syndr 1993; 6: 1130-1138

2. Baum MK, Shor-Posner G. Lu Y, Rosner B, Sauberlich HE, Fletcher MA. Micronutrients and HIV-1 disease progression. AIDS 1995; 9: 1051-1056.

3. De Luis DA, Aller R, Bachiller P, Eiros Bouzas JM, Arranz M, MartÍn J, Izaola O, Romero E. Nutritional treatment for acquired immunodeficiency virus infection using a enterotropic peptide-based formula enriched with $\mathrm{n} 3$ fatty acids: a randomized prospective trial. Eur J Clin Nutrition 2001; 55; 1048-1052.

4. Heymsfield SB, Casper K. Improved equations for anthropometric measurements of midarm muscle area. Am J Clin Nutr 1982; 35: 680690.

5. Frisancho AR. News norms of upper limb fat and muscle areas for assessment of nutritional status. Am J Clin Nutr 1981; 34: 540-545.

6. Mataix J, Mañas M. Tablas de composición de alimentos españoles. $3^{\mathrm{a}}$ Ed. Ed: University of Granada, 1998.

7. Centers for Disease Control and Prevention: Revised classification system for HIV infection and expanded surveillance case definition for AIDS among adolescents and adults. MMWR 1992; 41 (RR-17): 1-19.

8. National Research Council. Recommended Dietary Allowances, food and Nutrition Board, U.S., National Academy of Sciences, National Research Council, 1989.

9. Shenk A. Resting energy expenditure, weight loss, and altered body composition in HIV infection. Nutrition 1996; 12: 595-601.

10. Chlebowski RT, Grosvenor MB, Bernhard NH, Morales LS, Bulcavage LM. Nutritional status, gastrointestinal dysfunction, and survival in patients with AIDS. Am J Gastroenterol 1989; 84: 1288-1231.

11. Melchior JC, Niyongabo T, Henzel D, Durack-Bown I, Henri SC, Boulier A. Malnutrition and wasting, immunodepression, and chronic inflammation as independent predictors of survival in HIV-infected patients. Nutrition 1999; 15: 865-869.

12. Tang AM, Graham NM, Chandra RK, Saah AJ. Low serum vitamin B12 concentrations are associated with faster human immunideficiency virus type 1 disease progression. J Nutr 1997; 127: 345-351.

13. Shabert JK, Winslow C, Lacey J, Wilmore DW. Glutamine-antioxidant supplementation increases body cell mass in AIDS patients with weight loss: A randomized, double blind controlled trial. Nutrition 1999: 15: 860-864.

14. Endres S, Ghorbani R, Keley VE. The effect of dietary supplementation with n-3 polyunsaturated fatty acids on the syntesis of interleukin-1 and TNf alpha by mononuclear cells. N Engl J Med 1989; 320: 265-268.

15. de Luis DA, Aller R, Bachiller P, González Sagrado M, de Luis J, Izaola O, Terroba MC, Cuéllar L. Consejo nutricional aislado frente a suplemento y consejos nutricionales en pacientes con infección por VIH. Med Clin (Barc) 2003; 120: 565-567.

16. de Luis DA, Bachiller P, Aller R, de Luis J, Izaola O, Terroba MC, Cuéllar L, González Sagrado M. Relation among micronutrient intakes with CD4 count in HIV infected patients. Nutr Hosp 2002; 17: 285-9.

17. Gómez Candela C, de Cos Blanco AI, Mateo R, Castro E, Lorenzo A, Polo R. Changes in carbohydrate metabolism in the HIV/AIDS patient. Nutr Hosp 2002; 17: 147-53.

18. Izquierdo Villarroyal B, Celaya Pérez S, Amiguet García JA. Diet survey and evaluation of ingested nutrients in a group of HIV patients]. Nutr Hosp 2002; 17: 97-106. 